\title{
Case Study: Changing Behaviours to Improve Documentation and Optimize Hospital Revenue*
}

\section{Margaret Oldfield}

Resource Utilization Nurse, Hôtel-Dieu Grace Hospital

Windsor, ON

\begin{abstract}
A deficit situation prompted Hôtel-Dieu Grace Hospital in Windsor, Ontario to examine aspects of the funding process. A review of charts suggested that the documentation in the patient record did not accurately reflect the care given. Improving the documentation could potentially improve hospital revenue.
\end{abstract}

This approach has been successfully used in the US, but is not reported to have been implemented widely within the Canadian setting. Coached by an American team, three experienced nurses were trained to work with physicians to explore opportunities to improve their documentation, and to work with the Health Records Technicians to optimize the accuracy of their efforts.

Results demonstrate that such an initiative could impact behavioural changes in the hospital setting, and that such changes could impact hospital revenue. Realizing that front-line staff could actively participate in financial recovery was very empowering.

\section{Introduction}

Hospital funding in Ontario is based on information sent to the Canadian Institutes of Health Information (CIHI). The information details the diagnosis that is most responsible for the patient's hospital stay, other conditions or prob-

\footnotetext{
* Originally published in the Longwoods Case Study Library, 2006, www.longwoods.com/home. php?cat $=140$
} 
lems that were present prior to admission and those events or diagnoses that occurred during admission. The listing of the various conditions and disease pro cesses, as well as all of the interventional procedures done during the stay, provide a picture of how sick the patient has been and how much of the hospital resources were required in providing care. This information is entered into the health records coding program (ICD-10), and the case is assigned a main diagnostic category (CMG), a complexity level and a Relative Intensity Weighted value (RIW). Complexity levels range from 1 to 4 , with 1 representing a case that is uncomplicated, and 4 representing a case with numerous comorbidities and life-threatening conditions. The RIW increases with each level of complexity, and is also affected by the general age of the patient (Chu 1994). For most disease processes, the RIW is higher for patients in the geriatric population, reflecting the increased resources that are usually required to return a patient to pre-admission level of health. The RIW figure is the factor that determines the funding received for any patient. The funding system is not one where the hospital is reimbursed directly for the specific demands of each patient. The funding is based on the global picture of all of the patients who use the facilities over the course of the year, and so reflects the types of patient problems addressed in the hospital, and the general level of complexity or resource utilization represented by the patients. Generally, each 1.0 increase in RIW is associated with approximately $\$ 5,000.00$ in funding to the hospital.

Like many Ontario Hospitals, Windsor's Hôtel-Dieu Grace Hospital (HDGH) found itself in a deficit situation in recent years. Healthcare restructuring in the community, aimed at reducing four general hospitals to two, required the reconstruction of existing hospital sites. The associated costs of moving programs and relocating services to accommodate construction were major factors. Staffing was increased to prepare for new programs. At the same time, funding reductions were made, which contributed to the deficit situation. Hospital administration needed to look for ways to ensure that appropriate funding was being received for the care provided.

A retrospective review of the number of patients in the most frequently occurring diagnostic categories at HDGH showed that most of the patients were being coded at complexity levels 1 and 2, in spite of the general perception that HDGH patients are very ill. An American team of consultants was engaged to review charts for the quality of documentation and coding. The consultants suggested that there is opportunity for optimizing funding by improving the quality of the documentation in the patient record. It was pointed out that, for example, a postoperative patient may develop fever, elevated WBC count and purulent sputum. The physician would order appropriate antibiotics. These symptoms may be noted in the progress notes, but often a conclusion was not reached, such as pneumonia. When the health records technician (HRT) reviews and codes the chart, she 
cannot include pneumonia as a condition that occurred during the hospitalization. The development of pneumonia would impact the resources consumed by the patient, and most likely extend the length of stay. Without the statement of the diagnosis by the physician, the appropriate coding by the health records technician is not possible, and the revenue for having cared for this added condition is not realizable.

Ontario hospitals are not unique in this situation. Literature reports similar circumstances in American hospitals as well. An Accurate Coding Task Force was established at Midwest Orthopaedics and Rush-Presbyterian-St. Luke's Medical Center in Chicago. Cole et al. (1998) reported that the house staff was exposed to a one-hour instruction course, reviewing the issues and their impact on hospital reimbursement. Utilization Nurse/Quality Managers coached the physicians in the necessary changes. As a result, improved physician documentation leading to more accurate coding of the medical record was achieved. Increases in revenue were reported.

In a similar program at North Carolina Baptist Hospital, described by Hicks and Gentleman (2003), the documentation coordinators also helped to bridge the gap between the health records technicians and the physician. Doctors do not always know the terminology that is required for coding. The nurses bridged the gap between the clinical and coding worlds to obtain the documentation that supports the patient's severity of illness and intensity of service.

Staff at HDGH were convinced that the patients that were being cared for within our institution were much sicker than they appeared to be in reviewing CIHI data. As a result, HDGH launched a Documentation \& Coding Project with the following goals in mind:

1) to ensure that documentation reflects the "true acuity" of the patients cared for and

2) to ensure that the information forwarded to $\mathrm{CIHI}$ accurately represents the patient and the care received.

It was anticipated that increased accuracy of charting would be reflected in an increased number of patients coded at complexity levels 3 and 4, and that there would be an overall increase in average RIW and funding.

\section{Methodology}

Three registered nurses, who had worked in the organization for a number of years, were selected to work as documentation coordinators for the project. The objective was for the nurses to work with small groups of physicians on a daily 
basis to coach them in exploring opportunities to make their charting reflect the care being given. The nurses reviewed charts on assigned units on a daily basis, and communicated either in person or by a written note to the physician, identifying observed opportunities for improved documentation. Once the initial group of physicians was consistently documenting appropriately with little intervention, the nurses would move on to the next group, until all physicians were involved.

Three physician groups were part of the initial phase: doctors in ICU, the neurology/neurosurgery group and the Hospitalists. These groups were selected because of their general readiness to embrace new initiatives and because they represented resource intensive areas of the hospital. HDGH is the Regional Trauma Centre and Regional Stroke Centre. The Hospitalists are general practitioners employed by the hospital to look after medical patients who do not have a family physician with active privileges. These physicians are responsible for approximately $80 \%$ of the medical patients admitted to our hospital.

Simple tenets were developed:

1) If the problem is significant enough to warrant an order for treatment or a diagnostic procedure, there should be a note about it in the progress notes.

2) List diagnoses, not symptoms. If there is uncertainty, a possible or probable diagnosis is preferred to nothing at all.

3) Avoid unusual abbreviations or symbols. It is essential that what is written in the chart is readable and understandable by others involved in the patient's care, as well as the health records technician who must code the chart accurately.

At the same time, the nurses also liaised with the health records technicians, to evaluate the quality of the coding that was determining the information being sent to CIHI. The nurses learned how to code in ICD-10, and then coded the charts of the patients on their units and compared them with the findings of the health records technicians. It became apparent that the numerous choices required by the ICD10 program made it difficult for the health records technicians because of limited knowledge about various aspects of patient care and intervention. The nurses took the health records technicians to the bedsides of patients in various units, and explained aspects of patient care that they had read about in the charting.

\section{Results}

The project required the commitment of time and resources, and so it was important to determine whether the efforts were achieving any measure of success. One documentation coordinator audited approximately 65 charts completed early in the project, and compared them with a similar number of charts from prior to the 
project, matching the patients by age and diagnosis. There has been an observed shift in level of complexity towards the higher levels following intervention as compared to pre-intervention (Figure 1). In addition, an increase in the RIW has been observed (Figure 2).

Figure 1. Comparison of numbers of patients at each complexity level pre- and post-intervention

\begin{tabular}{|c|c|c|}
\hline & \multicolumn{2}{|c|}{ Number of Patients } \\
\hline Complexity Level & Pre-intervention & Post-intervention \\
\hline 1.00 & 10 & 5 \\
\hline 2.00 & 13 & 5 \\
\hline 3.00 & 19 & 16 \\
\hline 4.00 & 20 & 44 \\
\hline
\end{tabular}

\section{Figure 2.}

Observed improvements in level of complexity and RIW for hospitalist and intensive care physicians

\begin{tabular}{|l|l|c|c|}
\hline \multirow{2}{*}{ Program } & Data & Pre-intervention & Post-intervention \\
\hline \multirow{2}{*}{ Intensive Care } & Average Level of Complexity & 2.68 & 3.19 \\
\cline { 2 - 4 } & Average RIW & 2.49 & 3.33 \\
\cline { 2 - 4 } & Average Level of Complexity & 2.86 & 3.60 \\
\cline { 2 - 4 } & Average RIW & 5.32 & 6.86 \\
\hline
\end{tabular}

Overall, the documentation in the charts done after exposure to the Documentation and Coding Project showed statistically significant higher levels of complexity than the charts done prior to the project. While the observed improvement of RIW during the project was not statistically significant, it does carry a financial impact. Every 1.0 increase in RIW is assumed to represent approximately $\$ 5,000.00$ in funding to the hospital, so the changes noted above suggest the possibility of achieving an additional $\$ 5,200.00$ per patient in funding. It was gratifying to note that improving documentation in the patient's chart could impact hospital funding.

To evaluate the accuracy of the coding, a second audit compared the coding of charts by the documentation coordinators, as compared with the coding done by the health records technicians. In $45 \%$ of the charts, the documentation coordina- 
tors and the health records technicians completely agree on the main diagnosis (CMG) and the RIW. In 35\% of the charts, both groups reached the same CMG, but not the same RIW. In a further $20 \%$, the groups arrived at different CMGs and RIW. This certainly adds to concerns about the quality of the data being transmitted to CIHI.

The average difference in RIW between what the documentation coordinators coded and what the HRTs coded is 0.5297 , which could be linked to potential increased hospital remuneration of $\$ 2648.62 /$ patient. Again, it was encouraging that there is a potential increase in hospital funding linked to improvements in the coding of the charts - above and beyond the benefits of improved documentation.

\section{Discussion}

The prospect of attempting to change physician behaviour was somewhat daunting at the outset. We were unable to offer physicians financial remuneration for their efforts, which has often proved to be an excellent motivator (Shortell 1991). The use of documentation coordinators to work as coaches mirrored the change agents and idea champions that have been successful in other situations (Landry and Sibbald 2002; Rai and Howard 1994; Rogers 1983). It was essential that these documentation coordinators be knowledgeable about the types of patients they were reviewing, and have the respect of the physicians with whom they were working. Concentrating on a small group of physicians at a time ensured that prompt feedback could be provided, optimizing likelihood of success (Smits et al. 2002). It also afforded the opportunity of making the instruction specific to the patients on their service, and the types of charting deficits that had been observed. It has been suggested that interpersonal communication between physicians is a significant factor influencing adoption of an innovation (Coleman et al. 1966; Fennel and Warnecke 1988; Greer 1984). A surprising development during the project was the impact of physicians interacting with each other and discussing the project and its potential. Competitive natures motivated many to make sure that the charts for their service were as good as or better than those of another service. Congratulatory cards with gold stars on them were sent to those who enthusiastically embraced the project. One doctor pinned his star to his shirt, and wore it all day. He took great pride in describing what he had done to merit the star, and had his picture featured in the hospital newsletter. Following this, physicians were approaching the documentation coordinators in the hallways, asking for information about what they needed to do to improve their charting. The response certainly exceeded expectations. 
Not all physicians greeted the project eagerly on an everyday basis.

Occasionally the physicians would view the requests for changes to their charting to be extra work, which they did not have time for. However, we found that acknowledging a physician's workload, and explaining the potential impact of writing a couple of words on a patient's chart resulted in compliance.

The results are based on audits conducted using charts from the early months of the project. Sample sizes are relatively small, and attempts at matching samples are difficult, as no two patients truly respond alike. Considering that, the results do indicate a trend toward better documentation, which is reflected in increased levels of complexity and higher RIWs. Another audit involving larger samples after the project is well-established might be more indicative of the true measure of success. The financial measure of success will not be known for some time.

Results of the review of coding with the health records staff were shared with the director and managers of that department. It appears that quality improvement strategies, such as regular inter-rating of charts by various HRTs, could be implemented to enhance the consistency of results among the staff. The differences observed between the coding done by the documentation coordinators and the health records technicians generally relate to one of three things:

Additional diagnoses found by the documentation coordinators were generally found in the progress notes. Frequently because of time constraints, the health records technicians would refer only to the Discharge Summary, History \& Physical, and Operative and Consult Reports.

The main diagnosis was assumed to be the admitting diagnosis, when, frequently, some other diagnosis may have been more responsible for the bulk of the hospitalization.

An underlying diagnosis of a chronic condition may have been listed as the main diagnosis, instead of the illness that required the acute care stay.

\section{Conclusion}

Unfortunately, the project ended sooner than planned, due to the need to reallocate resources. However, there seem to be several positive results in addition to those noted above. First, we learned that physician behaviour can be 
modified. Our physicians understand the necessity of accurate documentation and billing from their office settings, and they were eager to participate in whatever was necessary to ensure the financial viability of the hospital. The improved documentation has enhanced the communication among other healthcare team members, improving patient care, as other team members are more aware of the patient's condition and the medical plan of care. The improved documentation is also helping to move the institution along toward its goal of an integrated patient care record. Allied healthcare workers are overcoming their fear of making notations in the Progress Notes, which had previously been allocated for physicians only. The health records technicians have progressed in seeking out physicians or the documentation coordinators when they are unsure of the meaning of a particular notation in a chart. Above all else, the project has fostered respect for the role that each employee plays in the work that is done at HDGH. While the project has officially ended, many aspects of it are moving ahead. Physician groups that were not involved in the original efforts have requested information on how to improve their documentation. A request has been made to include instruction regarding documentation expectations in the orientation of new physicians as they join the staff. Informal coaching of individual physicians continues, with similar physician response to what was noted during the project. The project convinced staff that each of us can make changes in how we do our jobs that can positively impact the financial health of our workplace.

From all perspectives, the efforts were worthwhile. Those involved with the project would endorse similar initiatives at a later date, and would encourage their consideration in other institutions.

Correspondence may be addressed to: Margaret Oldfield, Resource Utilization Nurse, Hôtel-Dieu Grace Hospital, Windsor, ON; tel: (519)-973-4411 ext. 3008; email: poldfield@hdgh.org.

\section{References}

Chu, C. 1994. "Resource Intensity Weighing and Case Mix Grouping: Assumptions and Implications for Health Service Performance Evaluation.” Healthcare Management FORUM 7(1).

Cole, B.J., S. Fics and D.B. Levine. 1998. “Optimizing Hospital Reimbursement Through Physician Awareness: A Step Toward Better Patient Care.” Orthopedics 21(1): 79-83.

Coleman, J.S., E. Katz and H. Menzel. 1966. Medical Innovation: A Diffusion Study. Indianapolis, IN: Bobbs-Merrill. 
Fennel, M.L. and R.B. Warnecke. 1988. The Diffusion of Medical Innovations. New York: Plenum Press.

Greer, A.L. 1984. “Adoption of Medical Technology: The Hospital's Three Decision Systems.” International Journal of Technology Assessment in Health Care 1: 669-80.

Hicks, T.A. and C.A. Gentleman. 2003. "Improving Physician Documentation Through a Clinical Documentation Management Program.” Nursing Administration Quarterly 27(4): 285-89.

Landry, M.D. and W.J. Sibbald. 2002. "Changing Physician Behavior: A Review of Patient Safety in Critical Care Medicine.” Journal of Critical Care 17(20): 138-45.

Rai, A. and G.S. Howard. 1994. "Propagating CASE Usage for Software Development: An Empirical Investigation of Key Organizational Correlates.” Omega International Journal of Management-Science 22: 133-47.

Rogers, E.M. 1983. Diffusion of Innovations (3rd ed.). New York: Free Press.

Shortell, S.M. 1991. Effective Hospital-Physician Relationships. Ann Arbour: Health Administration Press.

Smits, P.B., J.H. Verbeek and C.D. DeBuisonje. 2002. "Problem Based Learning in Continuing Medical Education: A Review of Controlled Evaluation Studies.” BMJ 19: 153-56.

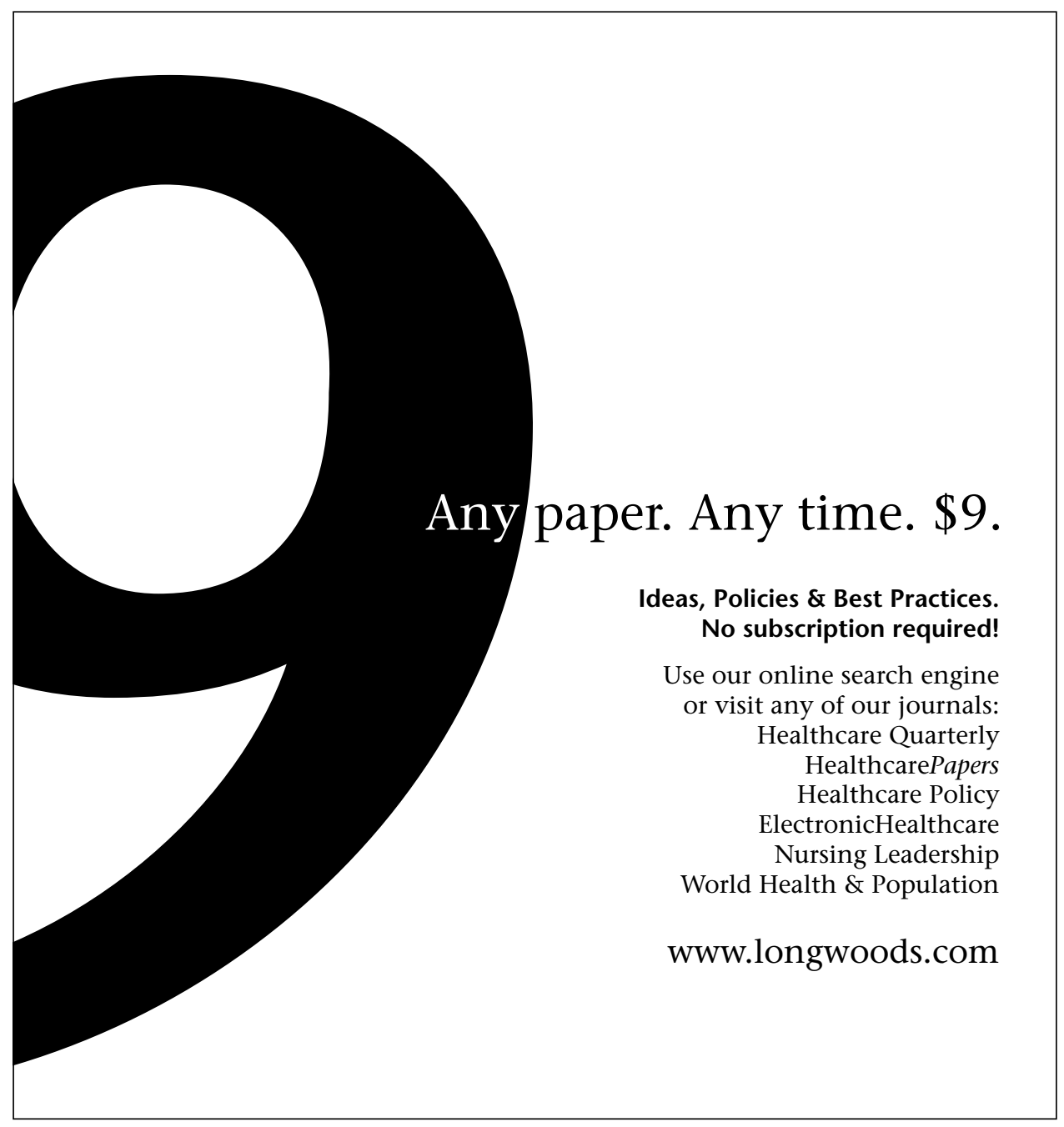

